\title{
Pre-Mixing and Masterbatch Approaches for Reinforcing Poly(Vinyl Acetate) With Cellulose Based Fillers
}

\author{
Apiradee Nicharat, ${ }^{a}$ Janak Sapkota $^{a \ddagger}$ and E. Johan Foster ${ }^{a, b *}$ \\ aAdolphe Merkle Institute, University of Fribourg, Chemin des Verdiers 4, CH-1700, Fribourg, \\ Switzerland

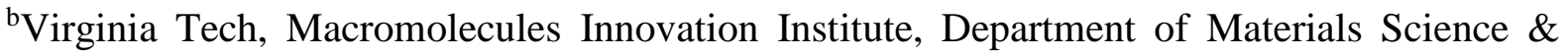 \\ Engineering, 445 Old Turner Street, 213 Holden Hall, Blacksburg VA 24061, USA \\ ‡ Authors contributed equally \\ Corresponding author: johanf@vt.edu
}

\begin{abstract}
Milling of microcrystalline cellulose (gMCC) and poly(vinyl acetate) (gPVAc) were performed, and the milled samples were pre-mixed prior to melt-mixing. Ground samples that were pre- and subsequently melt-mixed (gPVAc/gMCC) showed similar reinforcement (modulus of $\sim 3 \mathrm{GPa}$ at $25{ }^{\circ} \mathrm{C}, \sim 9 \mathrm{MPa}$ at $90{ }^{\circ} \mathrm{C}$ ) compared to the directly mixed composites containing cellulose nanocrystals isolated by acid hydrolysis from cotton (PVAc/CNC, (modulus of $\sim 3 \mathrm{GPa}$ at $25^{\circ} \mathrm{C}$ and $\sim 10 \mathrm{MPa}$ at $90{ }^{\circ} \mathrm{C}$ ). Highly concentrated masterbatches (up to $60 \mathrm{wt} \%$ of $\mathrm{gMCC}$ ), are shown for the first time with cellulosic filler. These additive concentrates can be first prepared using a solution casting approach, aiding in efficient transport, and then diluted by melt-mixing when needed. Using DMF and water as solvent for preparation, melt-processing schemes that are easier to scale-up and exploit industrially were evaluated in this work.
\end{abstract}

Keywords: Cellulose nanocrystal, Natural fibres, Masterbatch, Melt-processing, Nanocomposite

Introduction 
Cellulose nanomaterials or nanocellulose has been studied widely because of their superior reinforcing capability in nanocomposite applications, due to their renewable nature, high aspect ratio, high on-axis stiffness and therewith high reinforcing capability (Beck-Candanedo et al., 2005; Habibi et al., 2010; Lin and Dufresne, 2014; Moon et al., 2011). Cellulose nanofillers are obtained from several bio-sources including cotton (Dong et al., 1998), softwood (Filpponen and Argyropoulos, 2010), hardwood (Beck-Candanedo et al., 2005), bacterial cellulose (Araki and Kuga, 2001), tunicates (Anglès and Dufresne, 2000) and microcrystalline cellulose (MCC) (A. N. Frone, 2011; Bondeson et al., 2006; Capadona et al., 2009). Cellulose nanofillers are usually produced by acid treatments with strong acids, such as sulfuric (Dong et al., 1998), hydrochloric (Araki et al., 1998), phosphoric (Camarero Espinosa et al., 2013) and hydrobromic acids (Filpponen and Argyropoulos, 2010): dissolving away amorphous sections of cellulose while leaving highly crystalline regions. Combinations of acidic treatment and high-pressure homogenization have also been reported (M. Pan, 2013). Chemical or mechanical treatments of cellulosic material have shown to be efficient in producing nanometer or micrometer sized fillers with exceptional properties, resulting into a plethora of advanced applications (Eichhorn, 2011; Eichhorn et al., 2010; Habibi et al., 2010). However, the production of cellulose nanofillers by acidic treatment requires several neutralization steps that have slowed the mass-scale production of these nanofibers (Bandera et al., 2014; Camarero Espinosa et al., 2013; Capadona et al., 2007; Sapkota et al., 2013). Although several commercial plants producing $\mathrm{CNCs}$ have recently come online, $\mathrm{MCC}$ has proven to be an interesting alternative. A recent study has demonstrated the impact of different mechanical treatments on commercially available MCC to produce cellulose nanomaterials having similar reinforcement as of the commercially available CNCs (Bandera et al., 2014).

Homogenous dispersion of $\mathrm{CNCs}$ in nanocomposite materials is the primary requirement for effective reinforcement (Bandera et al., 2014; Sapkota et al., 2014). Casting / evaporation based 
processing techniques have been widely exploited, as they predominantly use hydrogen bonding solvents which facilitates the good dispersion of unmodified CNCs in the nanocomposites thus obtained (Nicharat et al., 2015; Rusli et al., 2010; Shanmuganathan et al., 2009). However, considering the amount of solvent and the time required to obtain the composites, the industrial scaling of casting/evaporation based processing techniques is limited. Eventually, either quick removal of solvent, and/or industrial processing techniques such as extrusion and injection molding will be preferred for large volume production (Nicharat et al., 2015; Sapkota et al., 2015).

Based on the previous studies, once CNCs are well dispersed in a poly(vinyl acetate) (PVAc) matrix (using solution casting), the resulting composite can be effectively re-processed in low shear melt-mixing conditions, such as those find in a roller-blade type mixer, allowing one to keep the morphology well preserved (Sapkota et al., 2015). When direct mixing PVAc granules with lyophilized CNCs in similar conditions, an effective dispersion of CNCs was predominantly observed, even though the as-obtained composites showed slightly lower reinforcement than the solution cast composites. Considering the need for an industrially viable approach, direct mixing is a more preferable mixing method, and therefore the addition of premixing techniques/steps prior to extrusion/melt-mixing could facilitate the preparation of wellmixed composites. Meanwhile, several studies have demonstrated the masterbatching approach, which involves dispersing a high concentration of nanofiller into a matrix for easier transport, with an end-user diluting the material using straight forward (industrially viable processing methods), as an effective approach to obtain nanocomposites with homogenous dispersion (Lepoittevin et al., 2003; Prashantha et al., 2009; Shah and Paul, 2004). More recently, Mariano et al. (Mariano et al., 2015) showed the possibility of using highly concentrated CNC / polycarbonate (PC) composites, and subsequently diluting these composites by extrusion. In this approach, prior to melt-mixing, CNCs were coated with PC, 
using a dissolution/precipitation process in which an aqueous dispersion of CNCs had the water exchanged to PC solubilizing pyridine. Even though the approach is quite interesting, it involves additional costs and scaling of a prohibitive solvent such as pyridine, what potentially limits the use of this technique in industrial processes.

To balance the high dispersibility advantage of lab-scale solution based approach and the need of an industrially viable processing technique, we implement a masterbatching approach that allows one to have highly concentrated CNC nanocomposites which when diluted via meltprocessing techniques such as compounding with roller blade mixer (RBM), maintain a high level of dispersion with virtually indistinguishable mechanical properties compared to the solution cast reference composites.

\section{Materials and Methods}

\section{Materials}

Poly (vinyl acetate) (PVAc, weight-average molecular weight, $\mathrm{Mw}=120,000 \mathrm{~g} / \mathrm{mol}$ ) was purchased from Anhui Herrman Impex Co., (China) and all reagents were purchased from Sigma Aldrich and were used without further purification. Microcrystalline cellulose (MCC) was purchased from FMC Biopolymers (Avicel Lattice NT-100). Cellulose nanocrystals (CNCs) were isolated from MCC via ultrasonication following earlier established protocols (Capadona et al., 2009). Briefly, $8 \mathrm{~g}$ of MCC were dispersed via ultrasonication in $400 \mathrm{~mL}$ of deionized water for 6 hours. The CNCs suspension was allowed to rest overnight, and the upper homogenously dispersed layer of the suspension was lyophilized using a VirTis BenchTop 2K $\mathrm{XL}$ lyophilizer with an initial temperature of $25^{\circ} \mathrm{C}$ and a condenser temperature of $-78^{\circ} \mathrm{C}$ to obtain dry CNCs.

\section{Preparation of ground PVAc (gPVAc) and MCC (gMCC)}


The dry milling of the PVAc (gPVAc) and MCC (gMCC) was performed using Hammer WittLAB (Frewitt SA, Fribourg, Switzerland) at hammer side using a screen 'Chevron' $0.2 \mathrm{~mm}$ for MCC and 0.35 for PVAc at $7000 \mathrm{rpm} .1 \mathrm{~kg}$ of PVAc or MCC was milled in 42 seconds cycle and consecutive cycles (11 for PVAc and 7 for MCC) were operated in order to obtain finer particles. To determine the milled particle size, Sieve analysis was performed using Retsch Analytical Sieve Shaker AS 200 Control for 30 g samples each with a vibration rate of 2 minutes, amplitude of $2 \mathrm{~mm} / \mathrm{g}$ and Vulkollan cubes as a sieving aid.

\section{Direct mixing of PVAc composites with MCC, gMCC and CNC using roller blade mixer (RBM)}

PVAc composites with $10 \mathrm{wt} \%$ filler content were prepared using direct mixing at low shear conditions in a laboratory-scale miniature batch mixer in a twin-cylinder configuration with two counters rotating roller blades (Brabender $\left.{ }^{\circledR} 30 \mathrm{EHT}\right)$ at $170{ }^{\circ} \mathrm{C}$ and $70 \mathrm{rpm}$. The polymer was first melted and subsequently MCC, gMCC and lyophilized CNCs were added to the molten PVAc. After mixing for 10 minutes, the composites were removed in the melt state from the mixing chamber and were allowed to cool at room temperature.

\section{Pre-mixing of gPVAc with MCC, gMCC and CNC prior to melt-mixing using roller blade mixer (RBM)}

gPVAc and the corresponding amount of filler $(10 \mathrm{wt} \%$ in the final composites in their dry state) were pre-mixed manually in powder state. The pre-mixed powder was fed in the low shear roller blade mixer and melt-mixed at $170{ }^{\circ} \mathrm{C}$ and $70 \mathrm{rpm}$ for 10 minutes. The composites were removed in the melt state from the mixing chamber and were allowed to cool at room temperature.

\section{Preparation of gPVAc/gMCC masterbatch composites by solution casting}

To prepare the masterbatch composites, two different solvents (dimethyl formamide (DMF) and water) were used as processing aid. gMCC was dispersed in DMF at a concentration of 20 
$\mathrm{mg} / \mathrm{mL}$ by sonication for $5-6 \mathrm{~h}$ in an ultrasonic bath (VMR TM USC600TH / 40kHz / 120W). gPVAc was dissolved in DMF at a concentration of $\sim 20 \% \mathrm{w} / \mathrm{w}$ by stirring for $4-6 \mathrm{~h}$. Aliquots of the gMCC dispersion and the gPVAc solution were combined in a flask and the mixtures were stirred for $1 \mathrm{hr}$ with a magnetic stir bar, sonicated for 1 hour and finally cast into Teflon ${ }^{\circledR}$ Petri dishes with a diameter of $12.7 \mathrm{~cm}$. The Petri dishes were placed into an oven at $70{ }^{\circ} \mathrm{C}$ for 3 to 4 days to evaporate most of the solvent and the resulting films were dried in a vacuum oven at a pressure of 400 mbar at $70^{\circ} \mathrm{C}$ for 2 days to remove any remaining solvent. Masterbatch composites containing 40-60 wt $\%$ of gMCC were thus produced. Similar approach was used with water as solvent to obtain similar composition.

\section{Dilution of gPVAc/gMCC masterbatch by roller blade mixer (RBM)}

gPVAc/gMCC masterbatch composites were diluted to gPVAc/gMCC $10 \mathrm{wt} \%$ composition using roller blade mixer (RBM). Briefly, appropriate parts of masterbatch composite were meltmixed with gPVAc powder directly in roller blade mixer at $170{ }^{\circ} \mathrm{C}$ and $70 \mathrm{rpm}$ for 10 minutes. For instance, to dilute gPVAc/gMCC $50 \mathrm{wt} \%$ masterbatch, $4 \mathrm{~g}$ of masterbatch composite was melt-mixed in RBM with $16 \mathrm{~g}$ of gPVAC under similar conditions as described above.

\section{Injection-molding of PVAc/CNCs nanocomposite films}

PVAc composites using the above methods, and shaped by injection molding (DSM Xplore micro-injection $10 \mathrm{cc}$ ). The materials made by the different methods were vacuum-dried for 24 $\mathrm{h}$ in a desiccator at room temperature and polymer was melt-injected to bar samples mold with dimensions $64 \times 12.7 \times 3.3 \mathrm{~mm}$ applying pressure of 10 bars.

\section{Morphological characterization}

The morphological analysis of MCC, CNC and gMCC were performed with transmission electron microscopy (TEM) on a Philips CM 100 operating at an accelerating voltage of $80 \mathrm{kV}$. For the sample preparation MCC, lyophilized CNCs and gMCC were dispersed in DMF at a 
concentration of $0.1 \mathrm{mg} / \mathrm{mL}$ by sonication in an ultrasonic bath (VMR USC600TH/40kHz/120W) for 10 minutes. Subsequently, 2-3 $\mu \mathrm{L}$ of the suspension were placed onto a carbon-coated copper grid. The samples were then dried at $70{ }^{\circ} \mathrm{C}$ in a vacuum oven for $2 \mathrm{~h}$ prior to analysis.

Scanning electron microscopy (Phenom Pro desktop, Phenom World, 62 PRO) was used to evaluate the morphology of gMCC, gPVAc and the composites. In order to probe the dispersion of filler in the composites, the composites were cryo-fractured and the fractured surface was analyzed with SEM. The acceleration voltage was set at $10 \mathrm{kV}$ and the surfaces were sputtercoated with $5 \mathrm{~nm}$ thick gold layer to avoid charging

\section{Dynamic mechanical analysis}

The dynamic mechanical properties of the composites were studied using a Q 800 dynamic mechanical analyzer (TA Instrument, Delaware, USA) in single-cantilever mode. Storage modulus were measured by temperature sweeps from 10 to $90{ }^{\circ} \mathrm{C}$ with a heating rate of 5 ${ }^{\circ} \mathrm{C} / \mathrm{min}$ and a constant frequency of $1 \mathrm{~Hz}$. At least 3 to 5 specimens were tested for each composition and the average values are presented.

\section{Results and discussion}

Here, we investigate the potential of using pre-mixing prior to direct melt-mixing and masterbatch-dilution approach to obtain composites that are feasible to process in an industrial environment. Masterbatching facilitates the preparation of highly concentrated filler (gMCC, MCC and CNC) content / polymer matrix (PVAc) dispersions, and these could easily be diluted afterwards in a low shear, melt-mixing (RBM) environment. The solution based processing methods are utilized in this study to obtain highly concentrated cellulose based fillers in the masterbatch composites. Even though solvent is used in this process, industrially viable water 
is compared to dimethylformamide (DMF), to better understand the engineering space available for processing. Several research articles have already shown that the solution casting mixtures of PVAc and CNCs from dimethylformamide and the subsequent evaporation of the solvent, affords nanocomposites with homogenously dispersed CNCs (Capadona et al., 2008; Nicharat et al., 2015; Rusli et al., 2010; Shanmuganathan et al., 2009). We extend a similar approach here to obtain masterbatch composites with up to $60 \mathrm{wt} \%$ cellulosic filler content. Even though the high filler content composites themselves do not present overly desirable properties, most likely due to aggregation caused by 'over-filling', they present an interesting opportunity for subsequent large scale dilution of these composites, in order to achieve composites with similar properties as of the pre-mixed and melt-mixed composites. Thus obtained highly concentrated masterbatches (up to $60 \mathrm{wt} \%$ filler content) were diluted to 10 wt $\%$ filler content using low shear roller blade mixer. Figure 1 shows the structure of starting materials and the morphology of CNC, MCC and milled components. The neat commercial beads of PVAc were milled down to an average particle size of $160 \pm 20 \mu \mathrm{m}$ as characterized by sieve analysis and also seen in optical microscope image (Figure 1 c). Similar approach was utilized to obtain milled MCC, to reduce the average particle size to $60 \pm 10 \mu \mathrm{m}$ (sieve analysis). The SEM images of the gMCC particles show aggregated particles, and on further analysis with TEM, the images show a similar microstructure like those of CNCs obtained from MCC (Figure 1). For gPVAc, the polymer molecular weight distribution remained unchanged as compared to the starting granule $\left(\mathrm{M}_{\mathrm{w}}=120,000 \mathrm{~g} / \mathrm{mol}\right)$.

\footnotetext{
*Insert Figure 1*
} 
The mechanical properties of the neat PVAc and filled composites made by directly mixing, masterbatch via solution cast and diluted composites were characterized by dynamic mechanical analysis (DMA) as a function of temperature and composition. The results are shown as plots of storage modulus $\left(E^{\prime}\right)$ as a function of temperature (Figure 2 and Figure 3 ). At room temperature $\left(25^{\circ} \mathrm{C}\right)$, the neat PVAc exhibits an $E^{\prime}$ of $\sim 2 \mathrm{GPa}$ (Figure 2a). Upon increasing the temperature, the material behavior transits from glassy to rubbery regime, where the $E^{\prime}$ drastically decreases to $3.6 \mathrm{MPa}\left(90^{\circ} \mathrm{C}\right)$. PVAc/CNC nanocomposites containing 10 wt $\%$ CNCs showed higher reinforcement compared to MCC or gMCC with significant increase in $E^{\prime}$ both at $25^{\circ} \mathrm{C}(\sim 3.0 \mathrm{GPa})$ and $90{ }^{\circ} \mathrm{C}(\sim 10 \mathrm{MPa})$. As expected in direct mixing, MCC and gMCC containing composite showed a moderate increase in $E^{\prime}\left(\sim 2.4 \mathrm{GPa}\right.$ at $25^{\circ} \mathrm{C}$ and $\sim 6 \mathrm{MPa}$ at $90{ }^{\circ} \mathrm{C}$ ). This disparity in reinforcing ability of different filler here is based on the type and morphology of the filler as evaluated in earlier studies(Capadona et al., 2008; Nicharat et al., 2015; Shanmuganathan et al., 2009, 2010). We surmise that CNCs shows better reinforcing effect in composites, predominately due to their high-aspect ratio, as compared to the filler particles based on MCC or gMCC.

*Insert Figure2*

Interestingly, when powder based pre-mixing is utilized prior to melt-mixing in low shear environment at RBM, a different behavior in mechanical reinforcement is observed. The reinforcement in thus obtained composites showed increased mechanical properties compared to the directly mixed composites. More specifically, the composites containing gPVAc and gMCC showed significantly higher reinforcement compared with all other samples, almost equaling the directly mixed PVAc/CNC composites within the temperature range both below 
and above the $\mathrm{T}_{\mathrm{g}}$ of the neat matrix. However, gPVAc/CNC composite showed increased $E^{\prime}$ at $25^{\circ} \mathrm{C}(\sim 3.2 \mathrm{GPa})$ and slightly lower reinforcement in higher temperature range (Figure $\mathbf{2 b}$ ). The higher reinforcement obtained when both ground particles are pre-mixed could be attributed to the enhanced dispersability gain as a result of matching particle size. The premixing of gPVAc and gMCC might have increased the mixing efficiency due to shear and diffusive mixing behavior, meaning the enhanced diffusion of gMCC particles into gPVAc during random mixing and melting conditions in the low shear mixing environment of RBM.

Figure 3a shows the $E^{\prime}$ as a function of temperature and composition for the masterbatch composites at different filler content (40-60 wt\%) formulated using a solution cast approach using either DMF or water as solvent. Depending on the processing approach and the composition, the modulus of the masterbatch composite seems to differ below $\mathrm{T}_{\mathrm{g}}$. For higher filler loading, the marginal improvement in the storage modulus shows that the mechanical behavior of the composites is governed by the aggregation of gMCC due to 'over-filling'. The increased concentration of gMCC in the masterbatch composites leads to an increase in hydrogen bonding between adjacent hydroxyls groups of gMCC, forming hydrogen bonds and causing aggregation. The aggregated $\mathrm{gMCC}$ will substantially reduce the specific surface area and the aspect ratio of the filler in the composite, which results in lower interface between gMCC and polymer matrix and hinders the effective stress transfer efficiency in the composite (Ma et al., 2015). Figure 3b shows the $E^{\prime}$ as a function of temperature and composition for the diluted composites, from $50 \mathrm{wt} \% \mathrm{gMCC}$ filler to $10 \mathrm{wt} \%$. Irrespective of the masterbatching approach used, the mechanical properties of the diluted composites are comparable to the PVAc/CNC directly mixed or gPVAc/gMCC premixed composites (within error).

\footnotetext{
* Insert Figure3*
} 
The morphology of the composites is studied with SEM and the homogeneous dispersion of the filler in the PVAc matrix can be clearly seen from the images. For PVAc/MCC composite (Figure 4a), some aggregates can be observed, but this could also be because the MCC particles were not fully exfoliated during the melt-mixing process. The gPVAc/gMCC composite did not show any significant aggregates, suggesting a more homogenous dispersion of the filler, more likely due to a better incorporation of gMCC into the gPVAc matrix, which could have been facilitated by the pre-mixing process, corroborating the improvement observed in the mechanical properties for this composite. In the highly concentrated masterbatch composites, it was expected that some aggregation would occur, due to increasing probability of adjacent hydroxyls forming hydrogen bonds, irrespective of the solvent used to prepare the composites. This can be clearly seen in the SEM images (Figure $4 \mathbf{c}$, d), with large voids representing the aggregated gMCC. The diluted composites show a homogenous filler dispersion with no significant aggregation, even though diluted composites from a water based masterbatch show more surface roughness compared to the masterbatch obtained with DMF as solvent, most probably due to PVAc being dissolved in DMF resulting in an even surface coating of gMCC. The masterbatch composite prepared by using water as solvent, might have resulted in a two phase system (similar to a latex), entrapping the filler between the polymer particles and hence ensuring the homogenous dispersion of the filler. 
*Insert Table 1* 


\section{Conclusions}

In summary, in this work, we showed the pre-mixing process as a viable approach to reinforce PVAc by using commercial MCC instead of CNCs, obtaining composites with similar or even enhanced mechanical properties. Using milling approach, we showed the facile process of obtaining homogenously mixed composite based on commercially obtainable gPVAc and gMCC. The composites thus obtained showed storage modulus comparable to those of PVAc/CNCs nanocomposites directly mixed in RBM. Also, we showed that it is possible to have highly concentrated masterbatch which when diluted results in composites that are similar to pre-mixed or directly mixed composites. DMF based masterbatch were used to evaluate the potential of water as solvent to prepare masterbatch, mainly because of the well established homogeneous dispersion of filler in DMF based solution approach. Even though the water did not dissolve PVAc, it was effective in maintaining homogenous dispersion of the diluted composites, most probably due to the system mimicking as a latex based suspension. Taken together, this shows the scalable approach for technological exploitation of cellulose based composites.

These approaches open up the possibility to develop melt-processing schemes that are easier to scale-up and utilize industrially. Considering the availability and commercialized nature of MCC, compared with the limited production of CNCs; milling of MCC could provide an industrially more relevant filler for upscaling the production of cellulose filled composites without compromising the mechanical reinforcement.

\section{Acknowledgements}

The authors gratefully acknowledge financial support from the Swiss National Science Foundation (NRP66: Resource Wood, Nr. 406640_136911/1), the Adolphe Merkle Foundation and the U.S. Endowment for Forestry and Communities ( $\left.\mathrm{P}^{3} \mathrm{Nano}\right)$. The Authors also thankfully 
acknowledge Christoph Weder for his support and scientific expertise. Author EJF is visiting

Professor in LabEx Tec 21, Grenoble, France (Investissements d'Avenir - grant agreement $\mathrm{n}^{\circ}$ ANR-11-LABX-0030).

\section{References}

A. N. Frone, D.P., D. Donescu, C. I. Spataru, C. Radovici, R. Trusca, R. Somoghi, 2011. BioResourches 6, 487-512.

Anglès, M.N., Dufresne, A., 2000. Plasticized Starch/Tunicin Whiskers Nanocomposites. 1. Structural Analysis. Macromolecules 33, 8344-8353.

Araki, J., Kuga, S., 2001. Effect of Trace Electrolyte on Liquid Crystal Type of Cellulose Microcrystals. Langmuir 17, 4493-4496.

Araki, J., Wada, M., Kuga, S., Okano, T., 1998. Flow properties of microcrystalline cellulose suspension prepared by acid treatment of native cellulose. Colloids and Surfaces A: Physicochemical and Engineering Aspects 142, 75-82.

Bandera, D., Sapkota, J., Josset, S., Weder, C., Tingaut, P., Gao, X., Foster, E.J., Zimmermann, T., 2014. Influence of mechanical treatments on the properties of cellulose nanofibers isolated from microcrystalline cellulose. Reactive and Functional Polymers 85, 134-141.

Beck-Candanedo, S., Roman, M., Gray, D.G., 2005. Effect of Reaction Conditions on the Properties and Behavior of Wood Cellulose Nanocrystal Suspensions. Biomacromolecules 6, 1048-1054.

Bondeson, D., Mathew, A., Oksman, K., 2006. Optimization of the isolation of nanocrystals from microcrystalline cellulose by acid hydrolysis. Cellulose 13, 171-180.

Camarero Espinosa, S., Kuhnt, T., Foster, E.J., Weder, C., 2013. Isolation of Thermally Stable Cellulose Nanocrystals by Phosphoric Acid Hydrolysis. Biomacromolecules 14, 1223-1230.

Capadona, J.R., Shanmuganathan, K., Trittschuh, S., Seidel, S., Rowan, S.J., Weder, C., 2009. Polymer Nanocomposites with Nanowhiskers Isolated from Microcrystalline Cellulose. Biomacromolecules 10, 712-716.

Capadona, J.R., Shanmuganathan, K., Tyler, D.J., Rowan, S.J., Weder, C., 2008. Stimuli-Responsive Polymer Nanocomposites Inspired by the Sea Cucumber Dermis. Science 319, 1370-1374.

Capadona, J.R., Van Den Berg, O., Capadona, L.A., Schroeter, M., Rowan, S.J., Tyler, D.J., Weder, C., 2007. A versatile approach for the processing of polymer nanocomposites with self-assembled nanofibre templates. Nat. Nanotechnol. 2, 765-769.

Dong, X., Revol, J.-F., Gray, D., 1998. Effect of microcrystallite preparation conditions on the formation of colloid crystals of cellulose. Cellulose 5, 19-32.

Eichhorn, S.J., 2011. Cellulose nanowhiskers: promising materials for advanced applications. Soft Matter 7, 303-315.

Eichhorn, S.J., Dufresne, A., Aranguren, M., Marcovich, N.E., Capadona, J.R., Rowan, S.J., Weder, C., Thielemans, W., Roman, M., Renneckar, S., Gindl, W., Veigel, S., Keckes, J., Yano, H., Abe, K., Nogi, M., Nakagaito, A.N., Mangalam, A., Simonsen, J., Benight, A.S., Bismarck, A., Berglund, L.A., Peijs, T., 2010. Review: current international research into cellulose nanofibres and nanocomposites. J. Mater. Sci. $45,1-33$.

Filpponen, I., Argyropoulos, D.S., 2010. Regular Linking of Cellulose Nanocrystals via Click Chemistry: Synthesis and Formation of Cellulose Nanoplatelet Gels. Biomacromolecules 11, 10601066.

Habibi, Y., Lucia, L.A., Rojas, O.J., 2010. Cellulose Nanocrystals: Chemistry, Self-Assembly, and Applications. Chemical Reviews 110, 3479-3500. 
Lepoittevin, B., Pantoustier, N., Devalckenaere, M., Alexandre, M., Calberg, C., Jérôme, R., Henrist, C., Rulmont, A., Dubois, P., 2003. Polymer/layered silicate nanocomposites by combined intercalative polymerization and melt intercalation: a masterbatch process. Polymer 44, 2033-2040.

Lin, N., Dufresne, A., 2014. Nanocellulose in biomedicine: Current status and future prospect. European Polymer Journal 59, 302-325.

M. Pan, X.Z., M. Chen 2013. BioResourches 8, 933-943.

Ma, L., Zhang, Y., Meng, Y., Anusonti-Inthra, P., Wang, S., 2015. Preparing cellulose nanocrystal/acrylonitrile-butadiene-styrene nanocomposites using the master-batch method. Carbohydr. Polym. 125, 352-359.

Mariano, M., El Kissi, N., Dufresne, A., 2015. Melt processing of cellulose nanocrystal reinforced polycarbonate from a masterbatch process. Eur. Polym. J. 69, 208-223.

Moon, R.J., Martini, A., Nairn, J., Simonsen, J., Youngblood, J., 2011. Cellulose nanomaterials review: structure, properties and nanocomposites. Chem. Soc. Rev. 40, 3941-3994.

Nicharat, A., Sapkota, J., Weder, C., Foster, E.J., 2015. Melt processing of polyamide 12 and cellulose nanocrystals nanocomposites. Journal of Applied Polymer Science 132, n/a-n/a.

Prashantha, K., Soulestin, J., Lacrampe, M.F., Krawczak, P., Dupin, G., Claes, M., 2009. Masterbatchbased multi-walled carbon nanotube filled polypropylene nanocomposites: Assessment of rheological and mechanical properties. Compos. Sci. Technol. 69, 1756-1763.

Rusli, R., Shanmuganathan, K., Rowan, S.J., Weder, C., Eichhorn, S.J., 2010. Stress-Transfer in Anisotropic and Environmentally Adaptive Cellulose Whisker Nanocomposites. Biomacromolecules $11,762-768$.

Sapkota, J., Jorfi, M., Weder, C., Foster, E.J., 2014. Reinforcing Poly(ethylene) with Cellulose Nanocrystals. Macromol. Rapid Commun. 35, 1747-1753.

Sapkota, J., Kumar, S., Weder, C., Foster, E.J., 2015. Influence of Processing Conditions on Properties of Poly (Vinyl acetate)/Cellulose Nanocrystal Nanocomposites. Macromol. Mater. Eng. 300, 562-571.

Sapkota, J., Roberts, M.N., Kumar, S., Weder, C., Foster, E.J., 2013. Melt-Processing of PVAcCellulose Nanocrystal Nanocomposites, in: Postek, M.T., Moon, R.J., Rudie, A.W., Bilodeau, M.A. (Eds.), Production and Applications of Cellulose Nanomaterials. TAPPI Press, pp. 111-112.

Shah, R.K., Paul, D.R., 2004. Nylon 6 nanocomposites prepared by a melt mixing masterbatch process. Polymer 45, 2991-3000.

Shanmuganathan, K., Capadona, J.R., Rowan, S.J., Weder, C., 2009. Stimuli-Responsive Mechanically Adaptive Polymer Nanocomposites. ACS Applied Materials \& Interfaces 2, 165-174.

Shanmuganathan, K., Capadona, J.R., Rowan, S.J., Weder, C., 2010. Bio-inspired mechanicallyadaptive nanocomposites derived from cotton cellulose whiskers. J. Mater. Chem. 20, 180-186. 

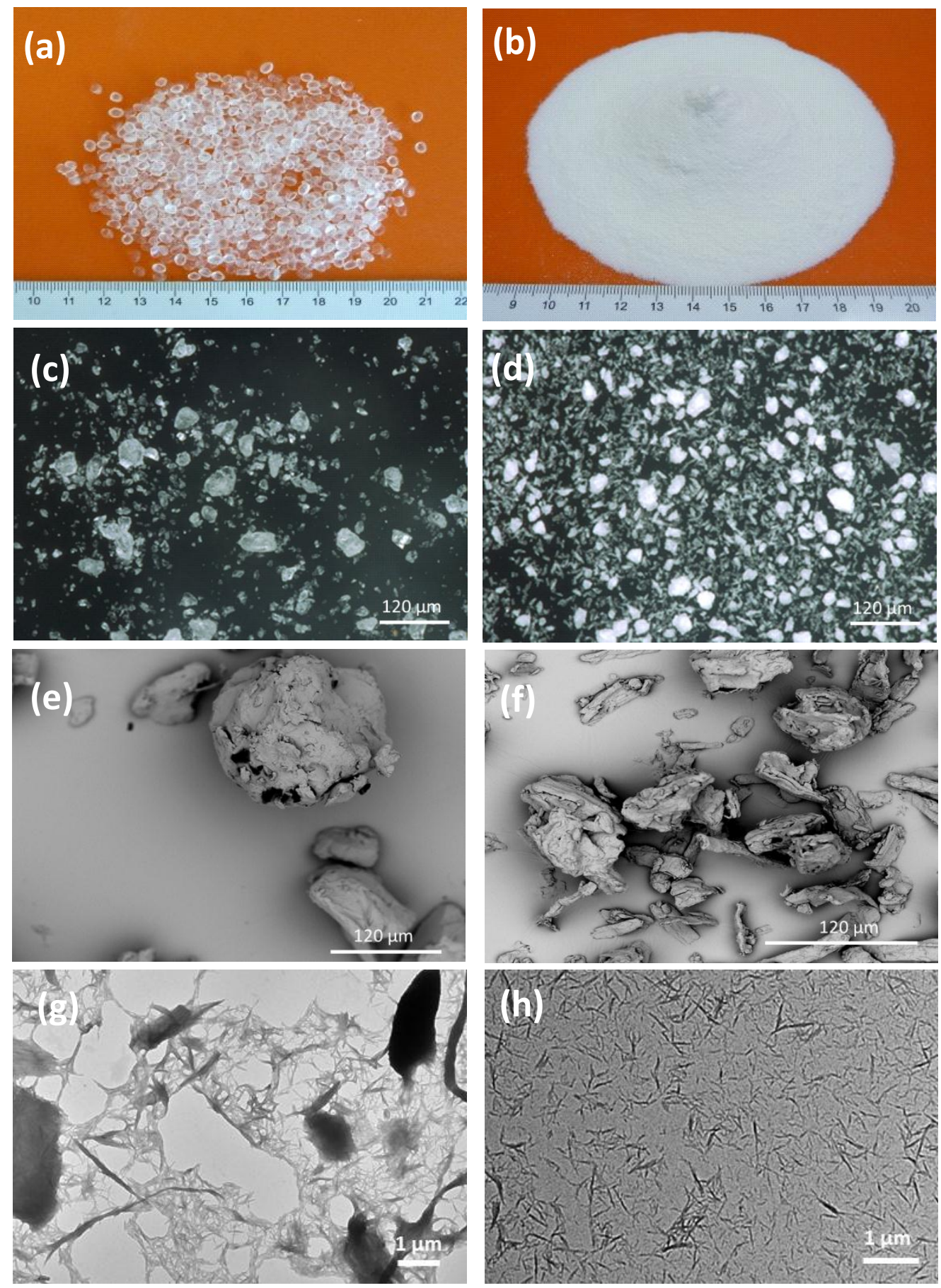

Figure 1. Photographs of (a) PVAc and (b) MCC (scale in $\mathrm{cm}$ ). Optical-microscope images of (c) gPVAc and (d) g-MCC. SEM images of (e) g-PVAc and (f) g-MCC. TEM image of (g) g$\mathrm{MCC}$ and (h) CNC. 
(a)

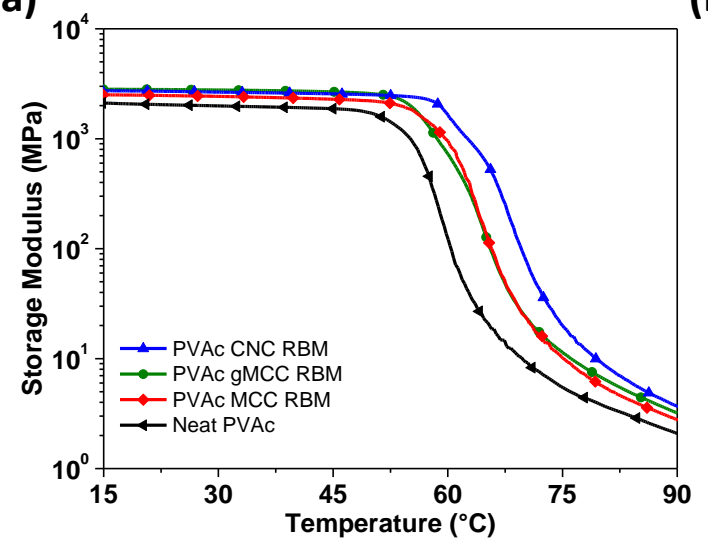

(b)

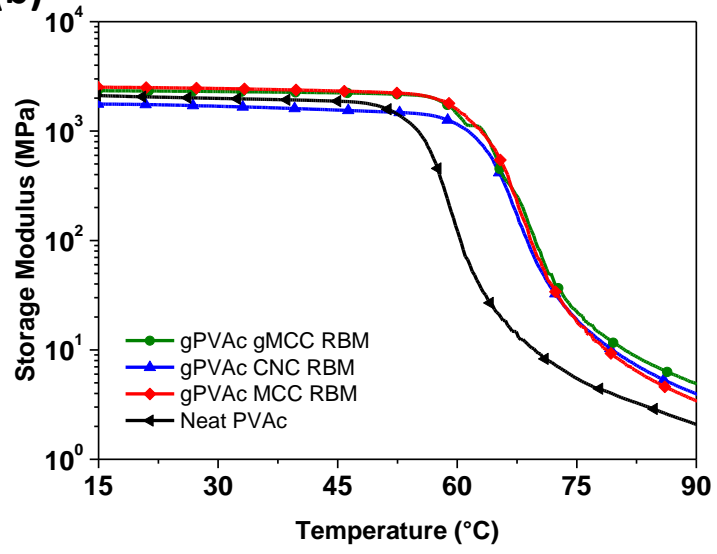

Figure 2. Dynamic Mechanical Analysis (DMA) traces of the neat PVAc and composites containing $10 \mathrm{wt} \%$ of MCC, gMCC or CNC prepared by a) directly melt-mixing and b) premixing and subsequently melt-mixing in a low-shear roller blade mixer environment.

(a)

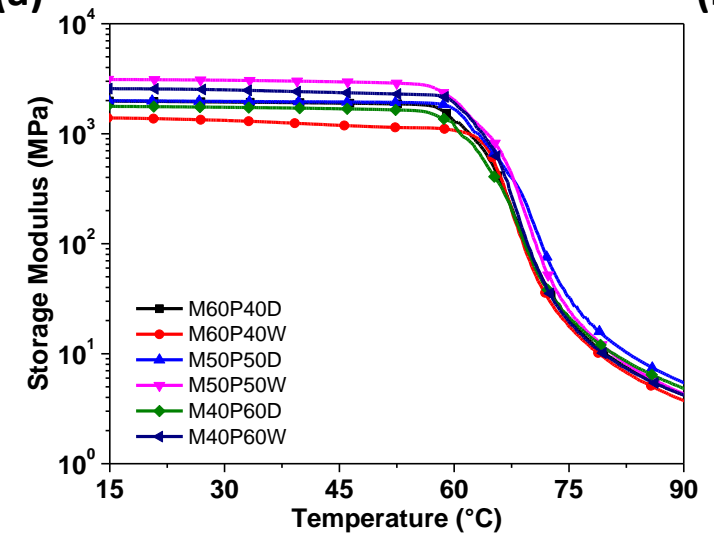

(b)

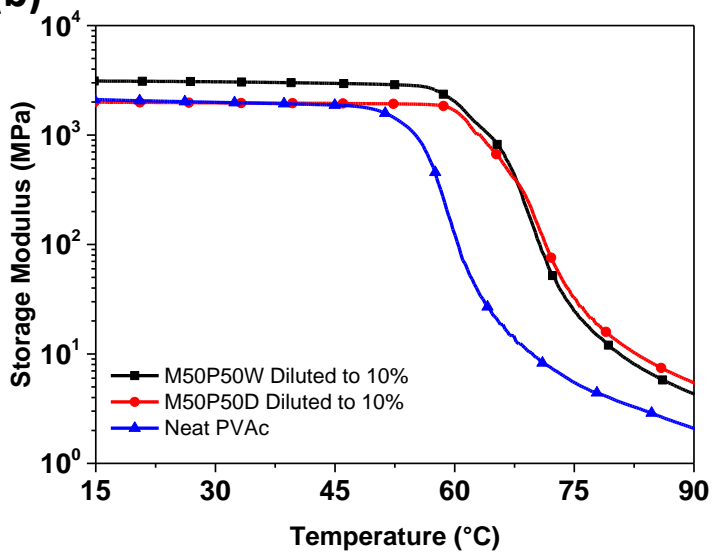

Figure 3. Dynamic mechanical thermal analysis traces showing tensile storage modulus $E^{\prime}$ as a function of temperature for masterbatch MxxPyy composites where $\mathrm{xx}$ and $\mathrm{yy}$ is the volume fraction of each component. MxxPyy concentrated masterbatch composites were prepared by solution cast using water (W) or DMF (D) as solvent and diluted to $10 \mathrm{wt} \%$ of gMCC via meltmixed. 

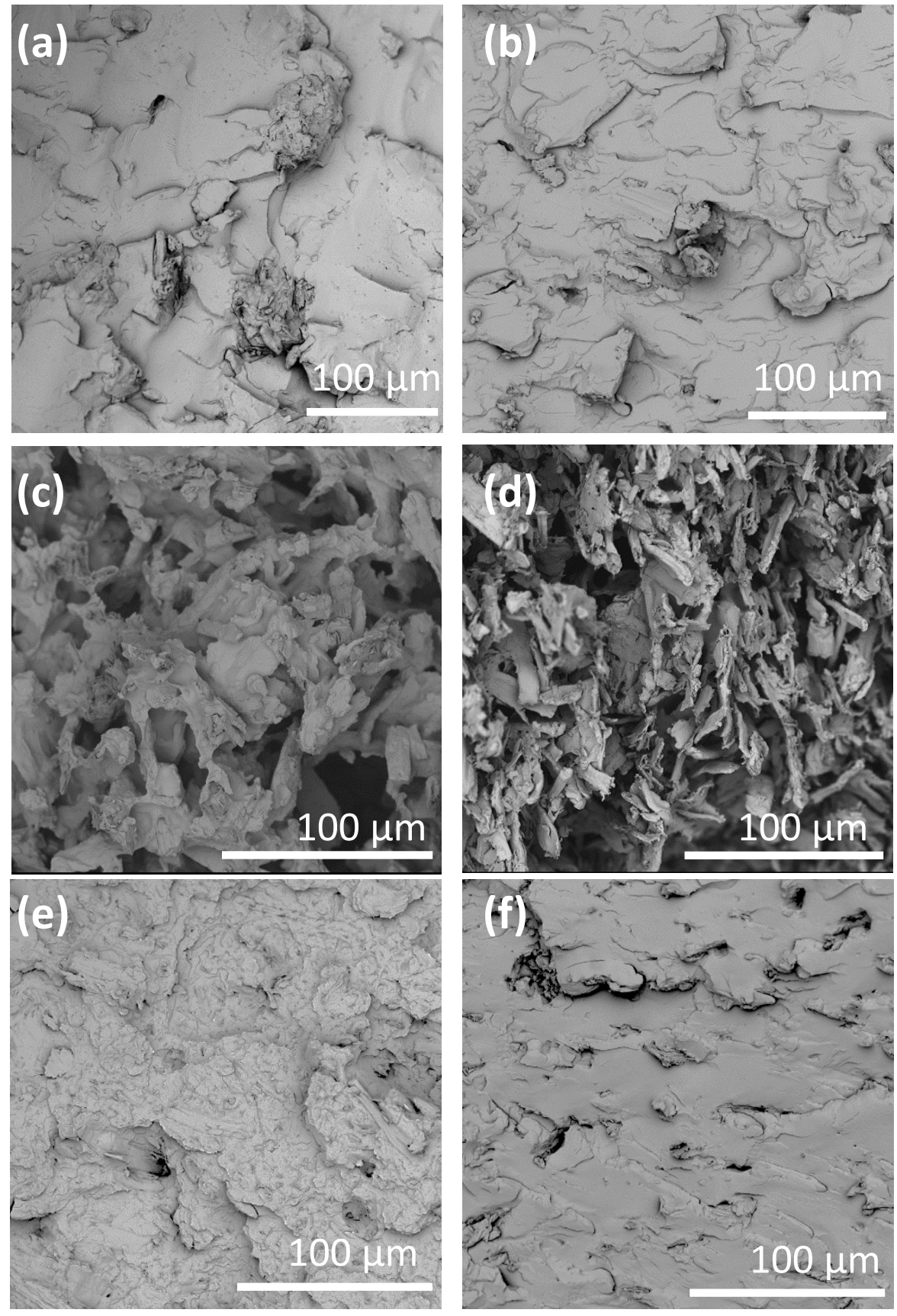

Figure 4. Scanning electron microscope images (a) PVAc/MCC $10 \mathrm{wt} \%$, (b) g-PVAc/g-MCC $10 \mathrm{wt} \%$, (c) masterbatch composite M50P50W (50 wt\%) (d) masterbatch composite M50P50D (50 wt\%) (e) diluted M50P50W composite to $10 \mathrm{wt} \%$ gMCC and (f) diluted M50P50D composite to $10 \mathrm{wt} \% \mathrm{gMCC}$. 
Table 1: Summary of mechanical properties of neat PVAc and composites

\begin{tabular}{|c|c|c|c|}
\hline Processing method & $\begin{array}{l}\text { Composition } \\
(10 \% \text { w/w filler } \\
\text { contents })\end{array}$ & $\begin{array}{l}\text { Storage } \\
\text { Modulus (MPa) } \\
\text { At } 25^{\circ} \mathrm{C}\end{array}$ & $\begin{array}{l}\text { Storage } \\
\text { Modulus (MPa) } \\
\text { At } 90^{\circ} \mathrm{C}\end{array}$ \\
\hline & Neat PVAc & $2000 \pm 60$ & $2.8 \pm 0.5$ \\
\hline \multirow{3}{*}{$\begin{array}{l}\text { Direct melt-mixing } \\
\text { in RBM }\end{array}$} & PVAc/MCC & $2455 \pm 110$ & $4.5 \pm 0.7$ \\
\hline & PVAc/gMCC & $2460 \pm 95$ & $5.8 \pm 0.9$ \\
\hline & $\mathrm{PVAc} / \mathrm{CNC}$ & $3095 \pm 80$ & $10.2 \pm 1.4$ \\
\hline \multirow{3}{*}{$\begin{array}{l}\text { Pre-mixing and } \\
\text { subsequent melt- } \\
\text { mixing in RBM }\end{array}$} & gPVAC/MCC & $2315 \pm 120$ & $5.8 \pm 1.0$ \\
\hline & gPVAc/gMCC & $2970 \pm 100$ & $8.8 \pm 1.2$ \\
\hline & gPVAc/CNC & $3210 \pm 115$ & $9.4 \pm 1.1$ \\
\hline \multirow{2}{*}{$\begin{array}{l}\text { Mastebatch-dilution } \\
\text { in RBM }\end{array}$} & M50P50W & $2990 \pm 130$ & $10.4 \pm 1.6$ \\
\hline & M50P50D & $3010 \pm 80$ & $8.3 \pm 1.9$ \\
\hline \multicolumn{4}{|c|}{$\begin{array}{l}\text { As determined by DMTA measurements, representing averages (number of } \\
\text { individual measurement, } \mathrm{N}=3-5 \text { ) } \pm \text { standard error measurements. }\end{array}$} \\
\hline
\end{tabular}

